\title{
"Cerrado" restoration by direct seeding: field establishment and initial growth of 75 trees, shrubs and grass species
}

\author{
Keiko Fueta Pellizzaro ${ }^{1,2}$ (D) Alba O. O. Cordeiro ${ }^{3} \cdot$ Monique Alves $^{1} \cdot$ \\ Camila P. Motta ${ }^{1}$ Gustavo M. Rezende ${ }^{1} \cdot$ Raissa R. P. Silva $^{4} \cdot$ José Felipe Ribeiro $^{5}$. \\ Alexandre B. Sampaio ${ }^{6}$ - Daniel L. M. Vieira ${ }^{1,7} \cdot$ Isabel B. Schmidt $^{1}$
}

Received: 11 October 2016/ Accepted: 14 February 2017/Published online: 4 March 2017

(C) Botanical Society of Sao Paulo 2017

\begin{abstract}
The coexistence of grasses, herbs, shrubs and trees characterizes savannas; therefore, to restore such ecosystems one should consider re-introducing all these growth forms. Currently, little is known about field establishment of most "Cerrado" (Brazilian savanna) species that could be used for restoration purposes. Most knowledge on restoration is focused on planting seedlings of tree species from forest physiognomies. Alternatively, direct seeding can be an appropriate method to re-introduce plants of different life forms to restore savannas. We evaluated the initial establishment success under field conditions of 75 "Cerrado" native species (50 trees, 13 shrubs, and 12 grasses) in direct seeding experiments in four sites in Central Brazil for 2.5 years. For that, we tagged and measured tree and larger shrub species and estimated ground cover by small shrub and grass species.
\end{abstract}

Electronic supplementary material The online version of this article (doi:10.1007/s40415-017-0371-6) contains supplementary material, which is available to authorized users.

Keiko Fueta Pellizzaro

keiko.pellizzaro@icmbio.gov.br; keikofueta@gmail.com

1 Programa de Pós-Graduação em Ecologia, Universidade de Brasília - UnB, Instituto de Biologia, Campus Darcy Ribeiro, Brasília, Distrito Federal 70919-970, Brasil

2 Instituto Chico Mendes de Conservação da Biodiversidade ICMBio, Reserva Biológica da Contagem, Rod. DF 003 Via EPIA km 8,5, Brasília, Distrito Federal 70635-800, Brasil

3 Programa de Pós-Graduação em Botânica, Universidade de Brasília - UnB, Instituto de Biologia, Campus Darcy Ribeiro, Brasília, Distrito Federal 70919-970, Brasil

4 Programa de Pós graduação em Ciências Florestais, UnB Fac. de Tecnologia - Secretaria de Pós-Graduação em Ciências Florestais, Campus Universitário Darcy Ribeiro, Brasília, Distrito Federal 70910-900, Brasil
Sixty-two species became established (42 trees, 11 shrubs and 9 grasses) under field conditions. Thirty-eight of the 48 tagged species had relatively high emergence rates $(>10 \%)$ and 41 had high seedling survival $(>60 \%)$ in the first year. Among grasses and small shrub species, Andropogon fastigiatus Sw., Aristida riparia Trin., Schizachyrium sanguineum (Retz.) Alston, Lepidaploa aurea (Mart. ex DC.) H.Rob., Stylosanthes capitata Vogel, S. macrocephala M.B.Ferreira \& Sousa Costa, Achyrocline satureioides (Lam.) DC. and Trachypogon spicatus (L.f.) Kuntze had the greatest initial establishment success (up to $30 \%$ soil cover). The data on harvesting period, processing mode and field establishment for these 75 species can be readily used in restoration efforts in the "Cerrado".

Keywords Direct sowing - Ecological restoration . Grassland restoration - Herbaceous layer - Neotropical savanna

5 Embrapa Cerrados, Empresa Brasileira de Pesquisa Agropecuária - Embrapa, Rodovia BR-020, Km 18 Caixa Postal: 08223, Planaltina, DF 73310-970, Brasil

6 Centro Nacional de Pesquisa e Conservação da Biodiversidade do Cerrado e Caatinga, CECAT/ICMBio, EQSW 103/104, Bloco “C”, Setor Sudoeste, Brasília, DF 70670-350, Brasil

7 Embrapa Recursos Genéticos e Biotecnologia CENARGEN, Parque Estação Biológica, PqEB, Av. W5 Norte (final) Caixa Postal 02372, Brasilia, DF 70770-917, Brasil 


\section{Introduction}

Savannas are naturally dominated by an herbaceous layer with tree density varying according to soil and climate conditions and fire regime, among other factors (Higgins et al. 2000). Therefore, ecological restoration of such areas must consider the original vegetation structure in order to actually contribute to conservation of biodiversity and ecosystem services (Chazdon 2008; Veldman et al. 2015a). Nevertheless, because most restoration studies are focused on forest ecosystems, restoration recommendations in both scientific and practical arenas are mostly focused on tree planting (Ruiz-Jaen and Aide 2005; Rodrigues et al. 2009). Afforestation or equivocal restoration threaten savanna and grassland ecosystems by decreasing endemic plant and animal diversity, decreasing ground water recharge and increasing aboveground biomass allocation, which increases susceptibility to fire events (Veldman et al. 2015b).

The dominance of exotic invasive species is a frequent challenge for restoring degraded ecosystems (Durigan et al. 2013; Holl et al. 2014). This is especially true in tropical savannas and grasslands, which are commonly dominated by invasive grasses (Williams and Baruch 2000). Invasive grass species reduce light and water availability (Levine et al. 2003); intensify fire regimes (D'Antonio and Vitousek 1992); and alter other ecosystem features (Chapin et al. 2000). Most grass species are shade-intolerant and can be eliminated by fast-growing forest trees in restoration areas, as long as fire and other disturbances are excluded (Cabin et al. 2002). However, planting fast-growing tree species that could outcompete these invasive grasses might not be possible or appropriate to restore grassland and savanna ecosystems (Veldman et al. 2015b). Besides, the seedlings from most native savanna tree species are slow-growing due to higher investment in below-ground tissues (De Castro and Kauffman 1998), which allows for survival during the dry season. In addition, native herbaceous and shrub species are important parts of open ecosystems structure, function and diversity (Mendonça et al. 2008; Bond and Parr 2010). Therefore, to effectively restore savanna and grassland environments, it is essential to select and use herbaceous and shrub species that can establish and compete with invasive grasses, without excluding slowgrowing tree species.

The "Cerrado" phytogeographical domain, in Central Brazil, is a biodiversity hotspot due to its high levels of endemism and high rates of conversion of native vegetation (da Silva and Bates 2002). It is the most biodiverse savanna region in the world, where millions hectares are targeted to be restored by federal legislation (Brasil 2000; Soares Filho et al. 2014). To effectively restore such vast areas, it is urgent to improve knowledge on restoration ecology, and the first step should be generating information on species propagation and establishment in field conditions. There are more than 12,000 plant species native from the "Cerrado" domain, many of which are endemic, and about 6000 are herbaceous (Ratter et al. 1997; Mendonça et al. 2008). Tree species diversity is high, especially in riparian forests, whereas herbs and shrubs represent $87 \%$ of the flora in the grassland and savanna physiognomies (Mendonça et al. 2008), which originally covered around $70 \%$ of the "Cerrado" domain (Sano et al. 2007). Native species from "Cerrado" grassland and savanna physiognomies, hereafter referred as "Cerrado", were rarely tested for field establishment (Silva et al. 2015), and very little is known about the use of herbaceous species for restoration in the Brazilian savanna (see Filgueiras and Fagg 2008; Aires et al. 2014). In the Federal District of Brazil, forest trees are often used to restore areas originally covered by "Cerrado", due to their faster growth rates, higher seed production and availability on nurseries (de Sousa 2015). This practice is also widespread across savanna ecosystems in the rest of Brazil.

Low-cost and effective methods are desirable for largescale restoration (Holl and Aide 2011; Campos Filho et al. 2013). Direct seeding is a relatively low-cost restoration technique that allows for the introduction of different plant growth forms simultaneously. While it is commonly applied worldwide in open ecosystems such as grasslands (Palma and Laurance 2015), restoration of savanna ecosystems in Brazil through direct seeding is still rare (Silva et al. 2015) and grassland restoration is almost nonexistent (Overbeck et al. 2013).

In this study, we aimed to investigate the establishment success in field conditions of a large number of species, of different growth forms, that could potentially be used in restoration experiments and practice. We present results of seedling emergence in both greenhouse and field conditions, as well as seedling survival in the field for 75 species (50 tree species, 13 shrubs and 12 grasses) native to "Cerrado" up to 2.5 years after seeding. Our results provide important information for species selection in restoration efforts in "Cerrado" areas.

\section{Methods}

Study sites - We evaluated the establishment success of 75 species seeded in seven restoration experiments in four sites in Central Brazil. Three study sites were located in the Federal District: (1) Água Limpa Experimental Farm of University of Brasília (15 $\left.56^{\prime} 55^{\prime \prime} \mathrm{S}, 47^{\circ} 56^{\prime} 03^{\prime \prime} \mathrm{W}\right)$; (2) Contagem Biological Reserve $\left(15^{\circ} 38^{\prime} 58^{\prime \prime} \mathrm{S}, 47^{\circ} 51^{\prime} 53^{\prime \prime} \mathrm{W}\right)$; (3) Entre Rios Farm $\left(15^{\circ} 57^{\prime} 30^{\prime \prime} \mathrm{S}, 47^{\circ} 27^{\prime} 26^{\prime \prime} \mathrm{W}\right)$, a private 
Table 1 Study sites, experimental and restoration areas through direct seeding of 75 savanna species in Central Brazil

\begin{tabular}{|c|c|c|c|c|c|c|c|c|}
\hline Site & $\begin{array}{l}\text { Altitude } \\
(\mathrm{m})\end{array}$ & $\begin{array}{l}\text { Annual rainfall } \\
(\mathrm{mm})\end{array}$ & $\begin{array}{l}\text { Year of } \\
\text { seeding }\end{array}$ & Soil type & $\begin{array}{l}\text { Restoration total } \\
\text { area }\left(\mathrm{m}^{2}\right)\end{array}$ & Experimental design & $\begin{array}{l}\text { Experimental } \\
\text { area }\end{array}$ & $\begin{array}{l}\text { Sampled } \\
\text { area }\end{array}$ \\
\hline 1 & 1080 & 1460 & $2011^{\mathrm{a}}$ & Latosol & 486 & $54 \times 6 \mathrm{~m} \times 1.2 \mathrm{~m}$ beds & $389 \mathrm{~m}^{2}$ & $389 \mathrm{~m}^{2}$ \\
\hline 2 & 1100 & 1668 & $\begin{array}{l}2012^{\mathrm{a}} \\
2013\end{array}$ & Latosol & $\begin{array}{l}30,000 \\
29,000\end{array}$ & $\begin{aligned} 36 & \times 30 \mathrm{~m} \text { rows } \\
6 & \times 20 \mathrm{~m} \times 20 \mathrm{~m} \text { plots }\end{aligned}$ & $\begin{array}{r}1080 \mathrm{~m} \\
2400 \mathrm{~m}^{2}\end{array}$ & $\begin{array}{l}1080 \mathrm{~m} \\
120 \mathrm{~m}^{2}\end{array}$ \\
\hline 3 & 1060 & 1350 & 2013 & Cambisol & 2400 & $6 \times 20 \mathrm{~m} \times 20 \mathrm{~m}$ plots & $2400 \mathrm{~m}^{2}$ & $120 \mathrm{~m}^{2}$ \\
\hline 4 & 1240 & 1453 & $\begin{array}{l}2012^{\mathrm{a}} \\
2013 \\
2014\end{array}$ & Latosol & $\begin{array}{l}30,000 \\
30,000 \\
30,000\end{array}$ & $\begin{array}{l}15 \times 10 \mathrm{~m} \times 100 \mathrm{~m} \text { plots } \\
12 \times 20 \mathrm{~m} \times 20 \mathrm{~m} \text { plots } \\
18 \times 20 \mathrm{~m} \times 20 \mathrm{~m} \text { plots }\end{array}$ & $\begin{array}{r}15,000 \mathrm{~m}^{2} \\
4800 \mathrm{~m}^{2} \\
7200 \mathrm{~m}^{2}\end{array}$ & $\begin{array}{l}135 \mathrm{~m}^{2} \\
240 \mathrm{~m}^{2} \\
360 \mathrm{~m}^{2}\end{array}$ \\
\hline
\end{tabular}

${ }^{a}$ We controlled weeds only on areas sown in 2011 and 2012 by manual weeding and/or mechanized mowing between beds, rows and plots

farm. Site 4, Chapada dos Veadeiros National Park $\left(14^{\circ} 07^{\prime} 03^{\prime \prime} \mathrm{S}, 47^{\circ} 38^{\prime} 31^{\prime \prime} \mathrm{W}\right)$, is located in the state of Goiás (Table 1).

All study sites were originally "Cerrado" sensu stricto areas that were converted to pasture. Only site 2 was used for mechanized agriculture, but it was colonized by exotic pasture grasses after abandonment. The study region is within a tropical savanna climate, with dry winters and rainy summers (Aw Köppen); the mean temperature is $21{ }^{\circ} \mathrm{C}$, and average precipitation is $1500 \mathrm{~mm}(90 \%$ of which is concentrated from October to May; INMET 2009). Mean precipitation in the four study sites is similar (Table 1).

Soils are latosols in sites 1, 2 and 4 and cambisols in site 3. All sites were dominated by invasive grass species (more than $98 \%$ soil cover), with very low density of native plants ( $<1$ individual, on average, per $10 \mathrm{~m}^{2}$ plot). Agricultural activities in all areas had been terminated before the start of restoration experiments. The most common invasive grasses in study sites are also common invaders throughout Brazil and other tropical areas (Zenni and Ziller 2011): Urochloa decumbens (Stapf) R.D. Webster, Urochloa humidicola (Rendle) Morrone \& Zuloaga, Urochloa brizantha (Hochst. ex A. Rich.) R.D. Webster, Andropogon gayanus Kunth, Melinis minutiflora P. Beauv. and Hyparhenia rufa (Nees) Stapf.

Experimental design - Direct seeding experiments were carried out from 2011 to 2014 according to the study sites (detailed in Table 1). We collected seeds/propagules used in the direct seeding experiments from areas around the restoration sites in the 8 months preceding the sowing, according to species phenology. We processed propagules according to each species features (detailed in Table 2). For species with seeds larger than $0.3 \mathrm{~cm}$, we selected and eliminated visually unviable seeds (predated, aborted). We stored seeds in paper bags in fresh (room temperature) and dry conditions until sowing. No pre-treatment to break seed dormancy was applied before seeding, except for Annona crassiflora Mart. seeds, which were soaked in a gibberellin acid solution (1 g of GA3, $200 \mathrm{~mL}$ of alcohol and $1 \mathrm{~L}$ of water) for $48 \mathrm{~h}$. We also used Stylosanthes spp. seeds sold commercially ( $S$. capitata and S. macrocephala), Campo Grande variety.

At all sites, soil was plowed one or two times during the dry season (May-October) prior to seeding to decrease dominance by invasive grasses and soil compaction. We carried out direct seeding manually at the beginning of the rainy season (late October-early December) following three field experiment types: sowing beds $(6 \times 1.2 \mathrm{~m})$; sowing rows (30 $\mathrm{m}$ linear meters); and broadcast sowing in whole plots $(20 \times 20 \mathrm{~m})$, according to year and experimental site (Table 1). We buried hard, large, round-seeded species $(\geq 0.5 \mathrm{~cm}$ diameter) by lightly plowing soil after seeding, whereas flat and smaller seeds were seeded after plowing on the soil surface (Table 2).

In sowing rows and beds, we planted one tree seed every $20 \mathrm{~cm}$ (one seed $\mathrm{m}^{-1}$ species ${ }^{-1}$ ). In seed-broadcasting plots, we sowed 25-34 tree seeds $\mathrm{m}^{-2}$ along with a mix of grass and shrub species in high density (4-16 species; seed density varying from 5 to 1100 viable seeds $\mathrm{m}^{-2}$ species $^{-1}$; Table 3). We chose this relatively high seed density to maximize the chances of promoting fast ground cover by native species and preventing the reestablishment and dominance of invasive grasses.

Data collection - To characterize seedling emergence during the first rainy season, we sampled experimental areas 3 and 6 months after sowing (which corresponds to the middle and the end of the first rainy season). To evaluate survival of woody species and ground cover of herbaceous species, we sampled the experimental areas every 6 months up to 2.5 years, which corresponds to the end of the second rainy season after seeding.

We tagged all seedlings from the 50 tree species and from eight of the shrub species in planting rows and beds, and measured their height (soil to apical bud) every 6 months. To sample seed-broadcasting experiments, we established two $10 \mathrm{~m}^{2}(20 \times 0.5 \mathrm{~m})$ subplots within each $400 \mathrm{~m}^{2}$ experimental plot. We estimated ground cover of native grasses and shrubs sowed by using the line-point intercept method (Herrick et al. 2009), sampling 200 points along a 20-m line 
Table 2 Growth form; seed collection time; processing mode (removing pulp device "RPD," sieve, grass shredder machine "GSM," manual separation "MS"); field planting mode (buried "B," or not buried "NB"); mean mass of 100 seeds \pm SD (values without SD were measured only once); number of seeds tested in green house $(\mathrm{GH})$ in each year $(\mathrm{Y})$; mean percentage seedling emergence in greenhouse (GHE) \pm SD (values without SD were tested only once-1 year) of Brazilian savanna native species

\begin{tabular}{|c|c|c|c|c|c|c|c|c|}
\hline Species & Family & $\begin{array}{l}\text { Growth } \\
\text { form }\end{array}$ & $\begin{array}{l}\text { Seed } \\
\text { collection }\end{array}$ & $\begin{array}{l}\text { Processing } \\
\text { mode }\end{array}$ & $\begin{array}{l}\text { Planting } \\
\text { mode }\end{array}$ & 100 seeds $(\mathrm{g})$ & $\begin{array}{l}\text { \# Seeds } \\
\text { GH: Y1; } \\
\text { Y2; Y3 }\end{array}$ & GHE $(\%)$ \\
\hline \multicolumn{9}{|l|}{ Grass species } \\
\hline $\begin{array}{l}\text { Andropogon bicornis } \\
\text { L. }\end{array}$ & Poaceae & Herb & May & GSM & NB & 0.23 & $300 ; 100$ & $5.5 \pm 6.0$ \\
\hline $\begin{array}{l}\text { Andropogon } \\
\text { fastigiatus } \mathrm{Sw} .\end{array}$ & Poaceae & Herb & June & GSM & NB & $0.11 \pm 0.01$ & 4000 & 0.0 \\
\hline Andropogon sp. & Poaceae & Herb & July & GSM & NB & 0.02 & $300 ; 200$ & $19.0 \pm 25.5$ \\
\hline Aristida riparia Trin & Poaceae & Herb & May & GSM & NB & $0.12 \pm 0.02$ & $\begin{array}{l}100 ; 100 \\
200\end{array}$ & $10.5 \pm 0.9$ \\
\hline Aristida aff. riparia. & Poaceae & Herb & June & GSM & NB & $0.15 \pm 0.03$ & $100 ; 4000$ & $1.5 \pm 2.1$ \\
\hline Aristida $\mathrm{sp} 1$ & Poaceae & Herb & May & GSM & NB & $0.15 \pm 0.02$ & 100 & 35.0 \\
\hline $\begin{array}{l}\text { Axonopus aureus } \\
\text { P.Beauv. }\end{array}$ & Poaceae & Herb & May & Sieve & NB & $0.06 \pm 0.01$ & na & na \\
\hline $\begin{array}{l}\text { Axonopus pellitus } \\
\text { (Nees ex Trin.) } \\
\text { Hitchc. \& Chase }\end{array}$ & Poaceae & Herb & May & GSM & NB & $0.012 \pm 0.008(\mathrm{~b})$ & 100 & 4.0 \\
\hline $\begin{array}{l}\text { Echinolaena inflexa } \\
\text { (Poir.) Chase }\end{array}$ & Poaceae & Herb & May & Sieve & NB & $0.22 \pm 0.04$ & 100 & 17.0 \\
\hline $\begin{array}{l}\text { Loudetiopsis } \\
\text { chrysothrix (Nees) } \\
\text { Conert }\end{array}$ & Poaceae & Herb & June & GSM & NB & $0.47 \pm 0.05$ & 4000 & 13.0 \\
\hline $\begin{array}{l}\text { Schizachyrium } \\
\text { sanguineum (Retz.) } \\
\text { Alston }\end{array}$ & Poaceae & Herb & June & GSM & NB & $0.19 \pm 0.05$ & $100 ; 4000$ & $3.5 \pm 0.7$ \\
\hline $\begin{array}{l}\text { Trachypogon } \\
\quad \text { spicatus } \text { (L.f.) } \\
\text { Kuntze }\end{array}$ & Poaceae & Herb & June & GSM & NB & 0.24 & $100 ; 4000$ & $2.5 \pm 3.54$ \\
\hline \multicolumn{9}{|l|}{ Shrub species } \\
\hline $\begin{array}{l}\text { Anacardium humile } \\
\text { A. St.-Hil. }\end{array}$ & Anacardiaceae & Shrub & $\begin{array}{l}\text { September- } \\
\text { October }\end{array}$ & MS & B & $238.39 \pm 7.60$ & $100 ; 100$ & $63.0 \pm 43.8$ \\
\hline $\begin{array}{l}\text { Achyrocline } \\
\text { satureioides (Lam.) } \\
\text { DC. }\end{array}$ & Asteraceae & Shrub & $\begin{array}{l}\text { August- } \\
\text { September }\end{array}$ & GSM & NB & $0.05 \pm 0.00$ & 100 & 4.0 \\
\hline $\begin{array}{l}\text { Aldama bracteata } \\
\text { (Gardner) } \\
\text { E.E.Schill. \& } \\
\text { Panero }\end{array}$ & Asteraceae & Shrub & April-May & GSM & NB & $0.15 \pm 0.04$ & $100 ; 100$ & $48.3 \pm 27.1$ \\
\hline $\begin{array}{l}\text { Lepidaploa aurea } \\
\text { (Mart. ex DC.) } \\
\text { H.Rob. }\end{array}$ & Asteraceae & Shrub & June-- & GSM & NB & $0.08 \pm 0.03$ & $100 ; 100$ & $10.5 \pm 0.7$ \\
\hline $\begin{array}{l}\text { Vernonanthura } \\
\text { phosphorica (Vell.) } \\
\text { H.Rob. }\end{array}$ & Asteraceae & Shrub & August & GSM & NB & $0.03 \pm 0.00$ & 100 & 10.0 \\
\hline $\begin{array}{l}\text { Jacaranda ulei } \\
\text { Bureau \& } \\
\text { K.Schum. }\end{array}$ & Bignoniaceae & Shrub & August & Sieve & NB & 2.70 (a) & 100 & 8.0 \\
\hline $\begin{array}{l}\text { Zeyheria montana } \\
\text { Mart. }\end{array}$ & Bignoniaceae & Shrub & August & Sieve & NB & 6.67 & 100 & 7.0 \\
\hline $\begin{array}{l}\text { Parinari obtusifolia } \\
\text { Hook.f. }\end{array}$ & Chrysobalanaceae & Shrub & October & RPD & $\mathrm{B}$ & 206.00 & 100 & 0.0 \\
\hline
\end{tabular}


Table 2 continued

\begin{tabular}{|c|c|c|c|c|c|c|c|c|}
\hline Species & Family & $\begin{array}{l}\text { Growth } \\
\text { form }\end{array}$ & $\begin{array}{l}\text { Seed } \\
\text { collection }\end{array}$ & $\begin{array}{l}\text { Processing } \\
\text { mode }\end{array}$ & $\begin{array}{l}\text { Planting } \\
\text { mode }\end{array}$ & 100 seeds $(\mathrm{g})$ & $\begin{array}{l}\text { \# Seeds } \\
\text { GH: Y1; } \\
\text { Y2; Y3 }\end{array}$ & GHE (\%) \\
\hline $\begin{array}{l}\text { Bauhinia cf dumosa } \\
\text { Benth. }\end{array}$ & Fabaceae & Shrub & October & Sieve & B & $372.00 \pm 114.00$ & 100 & $5.0 \pm 4.2$ \\
\hline $\begin{array}{l}\text { Mimosa claussenii } \\
\text { Benth. }\end{array}$ & Fabaceae & Shrub & September & GSM & B & $3.16 \pm 0.55$ & $\begin{array}{l}100 ; 100 \\
100\end{array}$ & $22.6 \pm 12.4$ \\
\hline Mimosa sp. & Fabaceae & Shrub & August & Sieve & $\mathrm{B}$ & $0.93 \pm 0.11$ & 100 & 0.0 \\
\hline $\begin{array}{l}\text { Senna alata (L.) } \\
\text { Roxb. }\end{array}$ & Fabaceae & Shrub & June & Sieve & B & $5.50 \pm 0.28$ & $100 ; 100$ & $13.0 \pm 9.5$ \\
\hline $\begin{array}{l}\text { Stylosanthes capitata } \\
\text { Vogel }+ \text { S. } \\
\text { macrocephala } \\
\text { M.B. Ferreira \& } \\
\text { Sousa Costa }^{\mathrm{a}}\end{array}$ & Fabaceae & Shrub & na & na & NB & $0.27 \pm 0.01$ & $100 ; 100$ & $23.3 \pm 4.6$ \\
\hline \multicolumn{9}{|l|}{ Tree species } \\
\hline $\begin{array}{l}\text { Anacardium } \\
\text { occidentale } \mathrm{L} .\end{array}$ & Anacardiaceae & Tree & $\begin{array}{l}\text { September- } \\
\text { October }\end{array}$ & MS & $\mathrm{N}$ & 448.43 & na & na \\
\hline $\begin{array}{l}\text { Astronium } \\
\text { fraxinifolium } \\
\text { Schott }\end{array}$ & Anacardiaceae & Tree & September & Sieve & $\mathrm{B}$ & $5.66 \pm 0.26$ & $100 ; 100$ & $79.3 \pm 20.3$ \\
\hline $\begin{array}{l}\text { Myracrodruon } \\
\text { urundeuva Allemão }\end{array}$ & Anacardiaceae & Tree & September & Sieve & B & $1.94 \pm 1.68$ & $\begin{array}{l}100 ; 100 \\
100\end{array}$ & $44.0 \pm 49.4$ \\
\hline $\begin{array}{l}\text { Schinopsis } \\
\quad \text { brasiliensis Engl. }\end{array}$ & Anacardiaceae & Tree & August & Sieve & B & $14.72 \pm 3.23$ & $100 ; 100$ & $4.5 \pm 4.9$ \\
\hline $\begin{array}{l}\text { Annona crassiflora } \\
\text { Mart. }\end{array}$ & Annonaceae & Tree & March & RPD & B & $64.95 \pm 5.95$ & $\begin{array}{l}100 ; 100 \\
100\end{array}$ & $26.0 \pm 24.0$ \\
\hline $\begin{array}{l}\text { Aspidosperma } \\
\text { macrocarpon Mart. }\end{array}$ & Apocynaceae & Tree & September & MS & $\mathrm{B}$ & $85.71 \pm 3.72$ & $100 ; 100$ & $40.0 \pm 32.9$ \\
\hline $\begin{array}{l}\text { Aspidosperma } \\
\text { tomentosum Mart. }\end{array}$ & Apocynaceae & Tree & September & MS & B & 21.6 & 100 & 46.0 \\
\hline $\begin{array}{l}\text { Hancornia speciosa } \\
\text { Gomes }\end{array}$ & Apocynaceae & Tree & October & RPD & B & $23.00 \pm 1.00$ & 100 & 63.0 \\
\hline $\begin{array}{l}\text { Schefflera } \\
\text { macrocarpa } \\
\text { (Cham. \& Schltdl.) } \\
\text { Frodin. }\end{array}$ & Araliaceae & Tree & July & Sieve & B & $5.88(\mathrm{e})$ & $100 ; 100$ & $20.0 \pm 24.0$ \\
\hline $\begin{array}{l}\text { Eremanthus } \\
\quad \text { glomerulatus Less. }\end{array}$ & Asteraceae & Tree & September & Sieve & NB & $0.40 \pm 0.19$ & $\begin{array}{l}200 ; 100 \\
100\end{array}$ & $32.0 \pm 40.9$ \\
\hline $\begin{array}{l}\text { Cybistax } \\
\text { antisyphilitica } \\
\text { (Mart.) Mart. }\end{array}$ & Bignoniaceae & Tree & October & MS & NB & $1.99 \pm 0.04$ & 200 & 49.0 \\
\hline $\begin{array}{l}\text { Handroanthus } \\
\text { ochraceus (Cham.) } \\
\text { Mattos }\end{array}$ & Bignoniaceae & Tree & $\begin{array}{l}\text { September- } \\
\text { October }\end{array}$ & Sieve & NB & $1.32 \pm 0.13$ & 100 & 93.0 \\
\hline $\begin{array}{l}\text { Jacaranda brasiliana } \\
\text { (Lam.) Pers. }\end{array}$ & Bignoniaceae & Tree & August & MS & B & $2.73 \pm 0.15$ & $\begin{array}{l}100 ; 100 \\
100\end{array}$ & $35.3 \pm 29.9$ \\
\hline $\begin{array}{l}\text { Tabebuia aurea } \\
\text { (Silva Manso) } \\
\text { Benth. \& Hook.f. } \\
\text { ex S.Moore }\end{array}$ & Bignoniaceae & Tree & $\begin{array}{l}\text { September- } \\
\text { October }\end{array}$ & Sieve & NB & 1.43 (a) & na & na \\
\hline $\begin{array}{l}\text { Tabebuia caraiba } \\
\text { (Mart.) Bureau }\end{array}$ & Bignoniaceae & Tree & October & MS & NB & $16.93 \pm 0.46$ & 100 & 39.0 \\
\hline $\begin{array}{l}\text { Cordia alliodora } \\
\quad \text { (Ruiz \& Pav.) Oken }\end{array}$ & Boraginaceae & Tree & October & Sieve & $\mathrm{B}$ & $7.67 \pm 0.13$ & 100 & 27.0 \\
\hline
\end{tabular}


Table 2 continued

\begin{tabular}{|c|c|c|c|c|c|c|c|c|}
\hline Species & Family & $\begin{array}{l}\text { Growth } \\
\text { form }\end{array}$ & $\begin{array}{l}\text { Seed } \\
\text { collection }\end{array}$ & $\begin{array}{l}\text { Processing } \\
\text { mode }\end{array}$ & $\begin{array}{l}\text { Planting } \\
\text { mode }\end{array}$ & 100 seeds $(\mathrm{g})$ & $\begin{array}{l}\text { \# Seeds } \\
\text { GH: Y1; } \\
\text { Y2; Y3 }\end{array}$ & GHE (\%) \\
\hline $\begin{array}{l}\text { Kielmeyera coriacea } \\
\text { Mart. \& Zucc. }\end{array}$ & Calophyllaceae & Tree & $\begin{array}{l}\text { August- } \\
\text { September }\end{array}$ & MS & NB & $10.85 \pm 0.25$ & $100 ; 100$ & $28.0 \pm 24.3$ \\
\hline Buchenavia sp. & Combretaceae & Tree & September & Sun-dried & B & $95.21 \pm 0.61$ & 100 & 0.0 \\
\hline $\begin{array}{l}\text { Buchenavia } \\
\text { tomentosa Eichler }\end{array}$ & Combretaceae & Tree & September & Sun-dried & $\mathrm{B}$ & $113.12 \pm 10.77$ & 100 & 30.0 \\
\hline $\begin{array}{l}\text { Terminalia argentea } \\
\text { Mart. }\end{array}$ & Combretaceae & Tree & September & Sieve & B & $24.96 \pm 0.37$ & 100 & 15.0 \\
\hline $\begin{array}{l}\text { Terminalia fagifolia } \\
\text { Mart. }\end{array}$ & Combretaceae & Tree & September & Sieve & $\mathrm{B}$ & 1.82 & 100 & 2.0 \\
\hline $\begin{array}{l}\text { Davilla elliptica } \\
\text { A.St.-Hil. }\end{array}$ & Dilleniaceae & Tree & August & GSM/sieve & B & 3.21 & 100 & 0.0 \\
\hline $\begin{array}{l}\text { Amburana cearensis } \\
\text { (Allemão) A.C.Sm. }\end{array}$ & Fabaceae & Tree & August & Sieve & B & 53.64 & $100 ; 100$ & $38.7 \pm 26.0$ \\
\hline $\begin{array}{l}\text { Anadenanthera } \\
\text { colubrina (Vell.) } \\
\text { Brenan }\end{array}$ & Fabaceae & Tree & August & Sieve & NB & $14.20 \pm 1.12$ & $\begin{array}{l}100 ; 100 \\
100\end{array}$ & $73.0 \pm 36.0$ \\
\hline $\begin{array}{l}\text { Bowdichia } \\
\quad \text { virgilioides Kunth }\end{array}$ & Fabaceae & Tree & July & MS & B & $2.12 \pm 0.02(\mathrm{c})$ & na & na \\
\hline $\begin{array}{l}\text { Copaifera } \\
\text { langsdorffii Desf. }\end{array}$ & Fabaceae & Tree & $\begin{array}{l}\text { August- } \\
\text { October }\end{array}$ & Sieve & B & $100.28 \pm 9.64$ & $\begin{array}{l}100 ; 100 \\
100\end{array}$ & $44.6 \pm 32.9$ \\
\hline $\begin{array}{l}\text { Dalbergia } \\
\text { miscolobium Benth. }\end{array}$ & Fabaceae & Tree & September & Sieve & $\mathrm{B}$ & $17.42 \pm 1.51$ & 100 & 12.0 \\
\hline $\begin{array}{l}\text { Dimorphandra mollis } \\
\text { Benth. }\end{array}$ & Fabaceae & Tree & June & GSM/sieve & $\mathrm{B}$ & $17.62 \pm 0.37$ & 100 & 6.0 \\
\hline Dipteryx alata Vogel & Fabaceae & Tree & September & None & B & $2259.06 \pm 48.47$ & $100 ; 100$ & $32.5 \pm 44.5$ \\
\hline $\begin{array}{l}\text { Enterolobium } \\
\text { contortisiliquum } \\
\text { (Vell.) Morong }\end{array}$ & Fabaceae & Tree & October & GSM/sieve & $\mathrm{B}$ & $45.31 \pm 0.81$ & $100 ; 100$ & $3.7 \pm 2.3$ \\
\hline $\begin{array}{l}\text { Enterolobium } \\
\text { gummiferum } \\
\text { (Mart.) J.F.Macbr. }\end{array}$ & Fabaceae & Tree & $\begin{array}{l}\text { July- } \\
\text { August }\end{array}$ & GSM & $\mathrm{B}$ & 51.02 & na & na \\
\hline $\begin{array}{l}\text { Hymenaea } \\
\text { stigonocarpa Mart. } \\
\text { ex Hayne }\end{array}$ & Fabaceae & Tree & September & GSM/sieve & $\mathrm{B}$ & $373.07 \pm 101.86$ & $\begin{array}{l}100 ; 100 \\
100\end{array}$ & $47.5 \pm 2.4$ \\
\hline $\begin{array}{l}\text { Machaerium opacum } \\
\text { Vogel }\end{array}$ & Fabaceae & Tree & August & Sieve & NB & $61.83 \pm 38.26$ & $100 ; 100$ & $3.6 \pm 3.1$ \\
\hline $\begin{array}{l}\text { Plathymenia } \\
\quad \text { reticulata Benth. }\end{array}$ & Fabaceae & Tree & August & Sieve & $\mathrm{B}$ & $4.50 \pm 0.06$ & 100 & 22.0 \\
\hline $\begin{array}{l}\text { Senegalia polyphylla } \\
\text { (DC.) Britton \& } \\
\text { Rose. }\end{array}$ & Fabaceae & Tree & July-ago & Sieve & $\mathrm{B}$ & $16.41 \pm 2.01$ & $100 ; 100$ & $55.7 \pm 25.5$ \\
\hline $\begin{array}{l}\text { Stryphnodendron } \\
\text { adstringens (Mart.) } \\
\text { Coville }\end{array}$ & Fabaceae & Tree & Ago & GSM/sieve & $\mathrm{B}$ & $9.40 \pm 0.52$ & $100 ; 200$ & $11.3 \pm 9.0$ \\
\hline $\begin{array}{l}\text { Tachigali vulgaris LF } \\
\text { Gomes da Silva \& } \\
\text { HC Lima }\end{array}$ & Fabaceae & Tree & September & Sieve & $\mathrm{B}$ & $22.02 \pm 0.50$ & $100 ; 100$ & $44.5 \pm 16.3$ \\
\hline $\begin{array}{l}\text { Vatairea macrocarpa } \\
\text { (Benth.) Ducke }\end{array}$ & Fabaceae & Tree & September & None & $\mathrm{B}$ & $142.86(\mathrm{~g})$ & $100 ; 200$ & $13.0 \pm 11.3$ \\
\hline $\begin{array}{r}\text { Emmotum nitens } \\
\text { (Benth.) Miers }\end{array}$ & Icacinaceae & Tree & November & None & $\mathrm{B}$ & $142.16 \pm 34.66(\mathrm{e})$ & na & na \\
\hline
\end{tabular}


Table 2 continued

\begin{tabular}{|c|c|c|c|c|c|c|c|c|}
\hline Species & Family & $\begin{array}{l}\text { Growth } \\
\text { form }\end{array}$ & $\begin{array}{l}\text { Seed } \\
\text { collection }\end{array}$ & $\begin{array}{l}\text { Processing } \\
\text { mode }\end{array}$ & $\begin{array}{l}\text { Planting } \\
\text { mode }\end{array}$ & 100 seeds $(\mathrm{g})$ & $\begin{array}{l}\text { \# Seeds } \\
\text { GH: Y1; } \\
\text { Y2; Y3 }\end{array}$ & GHE (\%) \\
\hline $\begin{array}{l}\text { Byrsonima } \\
\text { crassifolia }(\mathrm{L} .) \\
\text { Kunth }\end{array}$ & Malpighiaceae & Tree & April & RPD & B & $0.29 \pm 0.01(\mathrm{~d})$ & $100 ; 100$ & $24.7 \pm 14.4$ \\
\hline $\begin{array}{l}\text { Cecropia } \\
\text { pachystachya } \\
\text { Trécul }\end{array}$ & Urticaceae & Tree & $\begin{array}{l}\text { August- } \\
\text { September }\end{array}$ & MS & NB & $0.10 \pm 0.03$ & 100 & 0.02 \\
\hline $\begin{array}{l}\text { Eriotheca pubescens } \\
\text { (Mart. \& Zucc.) } \\
\text { Schott \& Endl. }\end{array}$ & Malvaceae & Tree & July & Sieve & $\mathrm{B}$ & $20.78 \pm 0.48$ & 100 & $33.5 \pm 7.8$ \\
\hline $\begin{array}{l}\text { Guazuma ulmifolia } \\
\text { Lam. }\end{array}$ & Malvaceae & Tree & October & GSM/sieve & B & 0.63 & 100 & 12.0 \\
\hline $\begin{array}{l}\text { Tibouchina } \\
\text { candolleana (Mart. } \\
\text { ex DC.) Cogn. }\end{array}$ & Melastomataceae & Tree & September & Sieve & NB & $0.11 \pm 0.07$ & $200 ; 100$ & $31.7 \pm 39.9$ \\
\hline $\begin{array}{l}\text { Brosimum } \\
\text { gaudichaudii } \\
\text { Trécul }\end{array}$ & Moraceae & Tree & October & MS & B & 142.86 & na & na \\
\hline $\begin{array}{l}\text { Eugenia dysenterica } \\
\text { (Mart.) DC. }\end{array}$ & Myrtaceae & Tree & October & RPD & B & $90.97 \pm 40.42$ & $100 ; 100$ & $8.0 \pm 6.9$ \\
\hline $\begin{array}{l}\text { Alibertia edulis } \\
\text { (Rich.) A.Rich. }\end{array}$ & Rubiaceae & Tree & $\begin{array}{l}\text { September- } \\
\text { November }\end{array}$ & Sieve & $\mathrm{B}$ & 0.89 (a) & 100 & 1.0 \\
\hline $\begin{array}{l}\text { Magonia pubescens } \\
\text { A. St.-Hil. }\end{array}$ & Sapindaceae & Tree & August & MS & NB & $182.32 \pm 55.15$ & $\begin{array}{l}100 ; 100 \\
100\end{array}$ & $62.0 \pm 38.0$ \\
\hline $\begin{array}{l}\text { Solanum lycocarpum } \\
\text { A. St.-Hil. }\end{array}$ & Solanaceae & Tree & $\begin{array}{l}\text { July- } \\
\text { December }\end{array}$ & RPD & $\mathrm{B}$ & $2.78 \pm 0.76$ & $\begin{array}{l}100 ; 100 \\
100\end{array}$ & $23.0 \pm 20.8$ \\
\hline $\begin{array}{l}\text { Qualea grandiflora } \\
\text { Mart. }\end{array}$ & Vochysiaceae & Tree & October & Sieve & NB & $12.0 \pm 4.0(\mathrm{f})$ & na & na \\
\hline
\end{tabular}

(a) Salomão et al. (2003), (b) Carmona et al. (1999), (c) Gonçalves et al. (2008), (d) García-Núnez et al. (2001), (e) Kuhlmann (2012), (f) Kutschenko (2009), (g) Mori et al. (2012)

na not available. Species are grouped by life form, listed in alphabetical order by family and species name

${ }^{\text {a }}$ Campo Grande variety, set of these two species sold commercially, evaluated as a sample

in each $10 \mathrm{~m}^{2}$ subplot (one point every $10 \mathrm{~cm}, 200$ points per subplot) every 6 months. We placed a 2-m-high stick straight up from the soil at each point and recorded the species touching the stick at the highest height; points with no plant species were recorded as bare soil.

Data analyses - We calculated seedling emergence percentage for 50 trees and eight of the shrub species by comparing the number of seedlings that emerged in the first rainy season (May-June) to the number of sowed seeds. We calculated the survival rates for the first year by comparing the number of plants surviving 12 months after sowing to the number of seedlings that emerged. We calculated the survival rate for the second year by comparing the number of plants still alive after 24 months to the number that survived the first year.
To verify the germinability of seeds used in field experiments, we also sowed seeds in a greenhouse simultaneously to each of the field experiments, except for the 2011 experiment. We distributed seeds of each species in plastic trays filled with subsoil lightly covering the seeds and irrigated daily. We monitored seedling emergence weekly for 16 weeks. For non-grass species, we planted 100 seeds species ${ }^{-1}$, except for species with low seed numbers. For grass species, we planted 4000 diaspores species $^{-1}$, due to small seed size and low germinability of native grasses (Table 1).

We tested a different group of species in each experiment; there was seeding density variation across experiments due to variations in site, year and seed availability. We do not intend to compare experiments, sowing methods 
Table 3 Grass and shrub species used in savanna direct seeding restoration experiments in three sites in Central Brazil

\begin{tabular}{|c|c|c|c|}
\hline Species & Sowing density $\left(\mathrm{g} \mathrm{m}^{-2}\right)$ & Soil cover after first rainy season & Soil cover after second rainy season \\
\hline Achyrocline satureioides & 0.880 & $0.28 \pm 0.37$ & $2.12 \pm 2.88$ \\
\hline Aldama bracteata & 0.033 & $0.12 \pm 0.18$ & $0.16 \pm 0.19$ \\
\hline Andropogon bicornis & 0.005 & $1.43 \pm 1.02$ & $0.04 \pm 0.38$ \\
\hline Andropogon fastigiatus & 0.500 & $30.24 \pm 3.79$ & na \\
\hline Aristida riparia ${ }^{\mathrm{a}, \mathrm{b}}$ & 0.100 & $1.21 \pm 1.21$ & $2.19 \pm 4.53$ \\
\hline Aristida riparia $^{\mathrm{a}}$ & 2.000 & $2.14 \pm 3.10$ & $15.06 \pm 12.08$ \\
\hline Axonopus aureus & 0.080 & $1.03 \pm 1.21$ & $0.47 \pm 1.41$ \\
\hline Axonopus cf. pellitus & 0.002 & $0.00 \pm 0.00$ & na \\
\hline Echinolaena inflexa & 0.100 & $0.75 \pm 0.67$ & $0.17 \pm 0.93$ \\
\hline Lepidaploa aurea $^{\mathrm{a}}$ & 0.900 & $7.43 \pm 9.00$ & $6.27 \pm 9.98$ \\
\hline Lepidaploa aurea ${ }^{\mathrm{a}}$ & 1.125 & $6.30 \pm 4.47$ & $21.25 \pm 11.93$ \\
\hline Loudetiopsis chrysothrix & 0.325 & $0.74 \pm 0.56$ & $0.20 \pm 1.82$ \\
\hline Schizachyrium sanguineum & 0.010 & $6.89 \pm 7.73$ & $15.95 \pm 18.47$ \\
\hline Stylosanthes spp. ${ }^{\mathrm{a}}$ & 0.060 & $2.80 \pm 3.10$ & $1.93 \pm 4.23$ \\
\hline Stylosanthes spp. ${ }^{\mathrm{a}}$ & 0.173 & $4.07 \pm 3.16$ & $3.77 \pm 3.07$ \\
\hline Trachypogon spicatus & 0.875 & $1.48 \pm 1.71$ & $2.28 \pm 6.86$ \\
\hline Vernonanthura phosphorica & 0.500 & $0.18 \pm 0.19$ & na \\
\hline
\end{tabular}

Sowing density (mean weight of seeds $/ \mathrm{m}^{2} \pm \mathrm{SD}$ ); soil cover (Mean $\pm \mathrm{SD}$ ) after first and second rainy season

na not available

${ }^{a}$ Species represented in more than one line were seeded in more than one experiment; each line represents the sowing density and consequent soil cover of each experiment

b Aristida sp. and Aristida aff. riparia were also seeded but had low rates of establishment, no flowering in the experimental areas and are not distinguishable from Aristida riparia in early stages, so data from these species establishment are not presented here

or even study years; therefore, no comparisons are presented for such purposes. The central aim of the analyses presented here is to synthesize information on seed harvesting period, processing and field establishment success of the studied species.

\section{Results}

In field conditions, 62 species (42 trees, 11 shrubs and 9 grasses) produced seedlings in the first rainy season after planting. Of these, 38 (32 trees and six shrubs) had at least $10 \%$ emergence in the first rainy season, with 30 of them (27 trees and three shrubs) reaching at least 20\%. After the first year, 36 trees and five shrubs had above $60 \%$ of survival with 19 of them (17 trees and two shrubs) having emergence above 20 and $>80 \%$ survival rate. Anacardium humile, Enterolobium gummiferum, Anacardium occidentale, Magonia pubescens, Handroanthus ochraceus and Vatairea macrocarpa were the species with best field establishment (see Table 4 and also Supplementary Material 1). The survival of woody individuals between the first and second year was in general similar to the one observed during the first year and relatively high for most species (Table 4).
After the first rainy season (6 months after sowing), tree seedling height was on average $7.2 \pm 5.9 \mathrm{~cm}$, and after the second rainy season (1.5 years after sowing) was $10.14 \pm 8.2 \mathrm{~cm}$. Tachigali vulgaris, Buchenavia tomentosa, Solanum lycocarpum, Plathymenia reticulata, Eremanthus glomerulatus and Hymenaea stigonocarpa were the fastest growing species (Table 4).

Among the grasses and shrub species evaluated by ground cover, Andropogon fastigiatus, Aristida riparia, Schizachyrium sanguineum, Lepidaploa aurea, Stylosanthes spp., Achyrocline satureioides and Trachypogon spicatus became best established in experimental areas, covering individually $2-30 \%$ of the soil. A. fastigiatus had the highest ground cover $(30 \%)$ in the first year after seeding, whereas other species tended to increase their ground cover in the second year, especially $A$. riparia, $L$. aurea and S. sanguineum (Supplementary Material 1). Most grass and small shrub species maintained similar ground cover between the first and second year after sowing (Table 3).

Most of the species germinated successfully in the greenhouse (62 species, Table 1), but nine of those species did not produce seedlings under field conditions (e.g., Byrsonima crassifolia, Cybistax antisyphilitica, A. crassiflora). Schefflera macrocarpa had a mean of at least $20 \%$ 


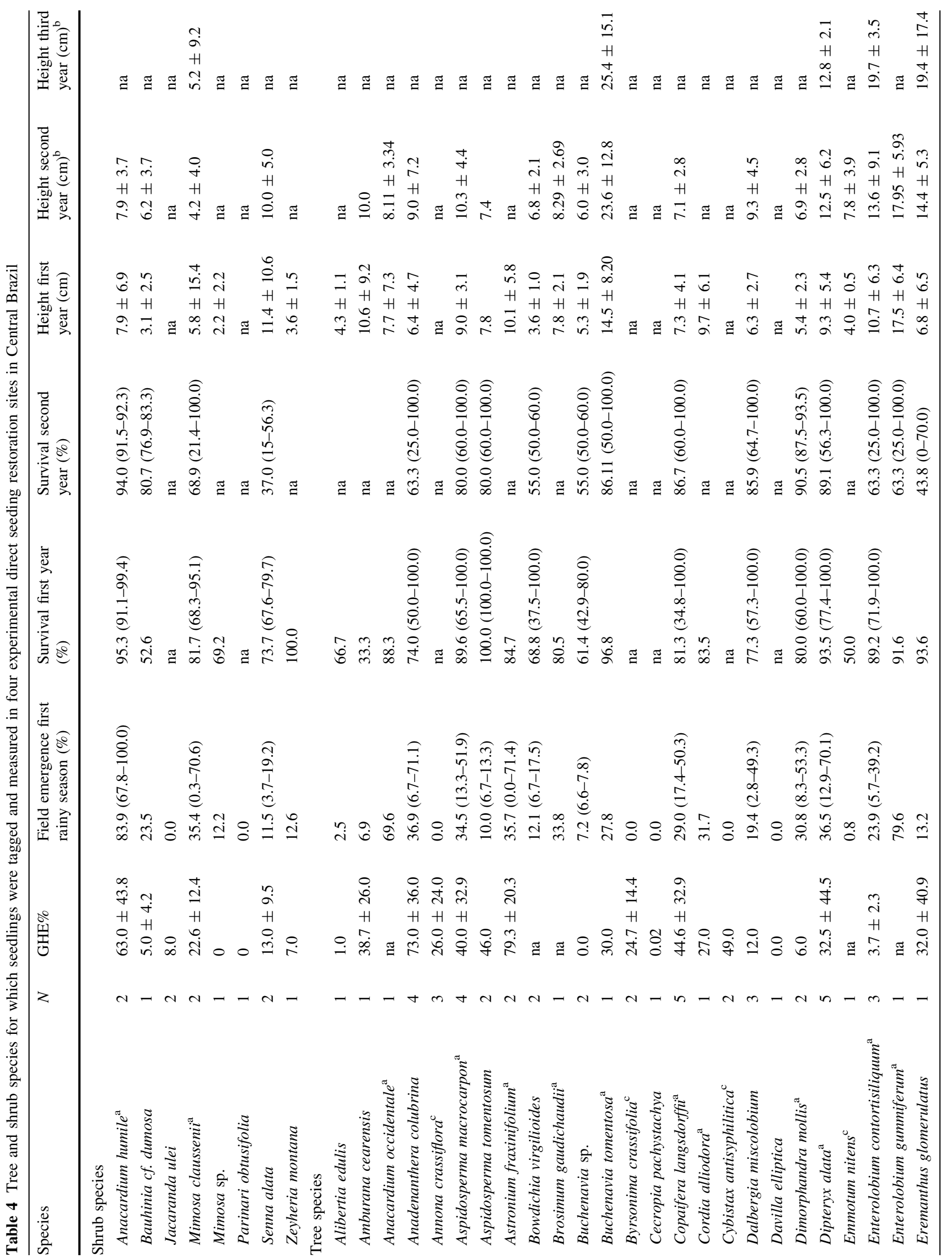




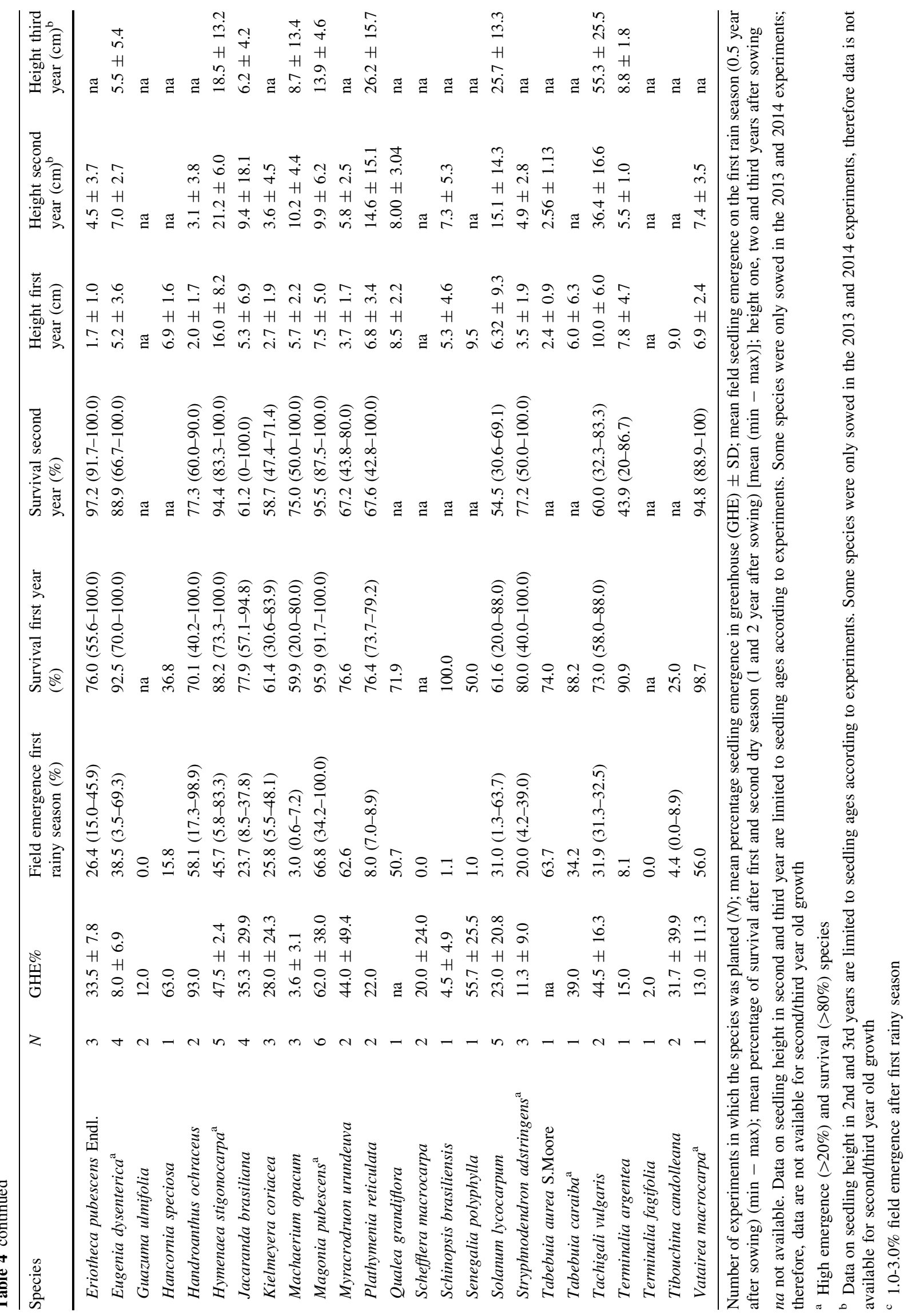


seedling emergence in the greenhouse and no emergence in field conditions. On the other hand, some species failed to germinate in greenhouse conditions but successfully established seedlings in the field (e.g., Mimosa sp. and Buchenavia sp., Table 4). Emergence in both the field and in greenhouse was in general higher for tree species compared to shrubs and grasses (Table 2).

\section{Discussion}

Our results suggest that through direct seeding, it was possible to promote the establishment, at least for the first 2.5 years, of 62 trees, shrubs and grass species in relatively large areas of "Cerrado" previously dominated by invasive grasses. The planting cost per individual seed in direct seeding restoration programs is low, and low rates of both emergence and survival rates are considered normal (Palma and Laurance 2015). Some authors consider a $10 \%$ emergence rate an acceptable threshold (Engel and Parrotta 2001; Campos Filho et al. 2013), and this value is near the mean emergence rate $(18 \%)$ obtained in most restoration projects around the world (Palma and Laurance 2015). We recorded 38 out of 58 woody species with at least a $10 \%$ emergence rate in the field; and identified 19 species with emergence rates above $20 \%$ associated with $\geq 80 \%$ survival rate after the first year. These results indicate that these species can be successfully used in restoration practices through direct seeding. In addition, even species with low establishment rates can be useful to help compose the community, and increase diversity and richness. Some of them should be included in direct seeding restoration programs especially when seed collection and storage are not expensive.

Aside from these species, we can infer that other naturally abundant native species with high seed production might be good candidates for use in direct seeding restoration practice. Our data from greenhouse experiments indicate that there might be no direct relationship between seedling emergence in a greenhouse and seedling establishment in field conditions. This suggests that greenhouse experiments might not be worth performing in order to select species suitable to be planted in direct seeding restoration programs. Some studied species had good field establishment rates, but low emergence in the greenhouse. In contrast, other species had high emergence rates in greenhouse conditions, but low establishment rate in the field. In a greenhouse, seeds can be sowed in a precise depth, on a flat soil without lumps, protected from predation, and there is no water shortage. However, in a greenhouse, high humidity of air and soil may increase seed infection by pathogens, and environmental triggers for germination such as thermal and humidity variations are absent.
We found high values of seedling survival (80\%) in the first 2.5 years, especially when compared to the $62 \%$ average survival of the seedling planting experiments for restoration identified in a recent review (Palma and Laurance 2015). Survival after the first dry season is a good parameter for long-term seedling establishment in savannas, where the length of the dry season can be a severe constraint to seedling survival due to water deficit in upper soil layers (Oliveira et al. 2005). Seedling survival between the first and second year was $92 \%$ on average for six "Cerrado" tree species in direct seeding experiments (Silva et al. 2015). For the 24 species for which we had survival data from the first to second year, survival rates varied from 54 to 97\% (Senna alata and Eriotheca pubescens, respectively) with a mean of $75 \%$. Seedlings' tolerance to drought may also allow these plants to survive extreme climatic events that might occur due to climate change (Palma and Laurance 2015). Aside from water deficit during the dry season, the major causes of sapling death were probably dry spells during the rainy season (Assad et al. 1993), competition with invasive grasses, and ant herbivory.

The slow growth of "Cerrado" tree seedlings observed here (see also Silva et al. 2015) is partly due to high investment in below-ground tissues (De Castro and Kauffman 1998; Hoffmann and Franco 2003). Due to the slow aboveground growth of savanna tree species, tree seedlings will be affected by invasive grasses for years. Also, trees in savannas will not shade the ground enough to control invasive grasses. Thus, a key strategy for the success of restoration in non-forest ecosystems is the introduction of fast-growing herbaceous species, in high density, that can cover the soil and compete with invasive grasses (Filgueiras and Fagg 2008; Hulvey and Zavaleta 2012). Although herbaceous species, especially grasses, tend to have low seed germinability, they mostly have high seed production. Therefore, seed harvesting can represent a low-cost strategy in some sites/regions, allowing for high density of seeding. Our data show that species such as $L$. aurea, A. riparia, A. fastigiatus, $S$. sanguineum, $T$. spicatus, Achyrocline satureoides and Stylosanthes spp., grew fast and showed high proportion of ground cover, and some species even reproduced in the first rainy season after planting. These plants may help to structure the community, allowing other native species to establish and survive; they assume a similar role of fast-growing tree species commonly recommended for restoration and invasive grasses control in forest ecosystems (Rodrigues et al. 2009). Native shrub and herbs can readily cover the ground, which can help control invasive grasses by the temporal priority effect (Young et al. 2001) and can affect invasive grasses productivity (Corbin and D'Antonio 2004) and dominance. 
This study presents information on a relatively large number of species, which represents a great increase in the otherwise scarce information on "Cerrado" species establishment in restoration areas, especially for herbaceous and shrub species. The information on fruiting period, fruit/ seed processing method and field establishment in early years after sowing for these species can contribute to the research and practice on ecological restoration of "Cerrado" areas. These results inform restoration allowing for actions that include the use of different growth forms and species diversity, which might potentially create a complex native community.

Acknowledgements These experiments were carried out under ICMBio research permit number 33390-3. We thank several students from Restaura-"Cerrado" research group for assistance; A. Alboyadjian for English review; Fundação Grupo o Boticário de Proteção à Natureza; ICMBio; CNPq (Edital MCT/CNPq/CT-Agro 26/2010); and Embrapa/CNA partnership of the Projeto Biomas for financial support.

\section{References}

Aires SS, Sato MN, Miranda HS (2014) Seed characterization and direct sowing of native grass species as a management tool. Grass Forage Sci 69:470-478

Assad ED, Sano EE, Masumoto R, De Castro LH, Da Silva FAM (1993) Veranicos Na Região Dos Cerrados Brasileiros Frequencia E Probabilidade de Ocorrência. Pesqui Agropecu Bras 28:993-1003

Bond WJ, Parr CL (2010) Beyond the forest edge: ecology, diversity and conservation of the grassy biomes. Biol Conserv 143:2395-2404

Brasil (2000) Lei 9.985, de 18 de Julho de 2000-Sistema Nacional de Unidades de Conservação. http://www.planalto.gov.br/ccivil_ 03/leis/L9985.htm. Accessed 21 Oct 2016

Cabin RJ et al (2002) Effects of light, alien grass, and native species additions on Hawaiian dry forest restoration. Ecol Appl 12:1595-1610

Campos Filho EM et al (2013) Mechanized direct-seeding of native forests in Xingu, Central Brazil. J Sustain Forest 32:702-727

Carmona R, Martins CR, Fávero AP (1999) Características de Sementes de Gramíneas Nativas Do Cerrado. Pesqui Agropec Bras 34:1067-1074

Chapin FS et al (2000) Consequences of CHANGING BIODIVERSITY. Nature 405:234-242

Chazdon RL (2008) Beyond deforestation: restoring forests and ecosystem services on degraded lands. Science 320:1458-1460

Corbin JD, D'Antonio CM (2004) Competition between native perenial and exotic annual grasses: implications for an historical invasion. Ecology 85:1273-1283

D'Antonio CM, Vitousek PM (1992) Biological invasions by exotic grasses, the grass-fire cycle, and global change. Annu Rev Ecol Syst 23:63-87

da Silva JMC, Bates JM (2002) Biogeographic patterns and conservation in the South American Cerrado: a tropical savanna hotspot. Bioscience 52:225

De Castro EA, Kauffman JB (1998) Ecosystem structure in the Brazilian Cerrado: a vegetation gradient of aboveground biomass, root mass and consumption by fire. J Trop Ecol $14: 263-283$

de Sousa AP (2015) Avaliação de Um Programa de Restauração de Uma Bacia Hidrográfica: Execução E Envolvimento Dos Proprietários. Dissertation, Universidade de Brasília, Brasília

Durigan G, Ivanauskas NM, Zakia MJB, De Abreu RCR (2013) Control of invasive plants: ecological and socioeconomic criteria for the decision making process. Nat Conserv 11:23-30

Engel VL, Parrotta JA (2001) An evaluation of direct seeding for reforestation of degraded lands in Central Sao Paulo State, Brazil. For Ecol Manag 152:169-181

Filgueiras TS, Fagg WC (2008) Gramineas Nativas Para Recuperação de Áreas Degradadas No Cerrado. In: Felfili CRMA, Sampaio JM, Correia JC (orgs.) Bases para a recuperação de áreas degradadas na bacia do São Francisco. Centro de Referência em Conservação da Natureza de Áreas degradadas (CRAD), Brasília, pp. 89-107

García-Núnez C, Azócar A, Silva JF (2001) Seed production and soil seed bank in three evergreen woody species from a neotropical savanna. J Trop Ecol 17:563-576

Gonçalves JVS, Albrecht JMF, Soares TS, Titon M (2008) Caracterização Física E Avaliação Da Pré-Embebição Na Germinação de Sementes de Sucupira-preta (Bowdichia virgilioides Kunth). Cerne Lavras 14:330-334

Herrick JE, Van Zee JW, Havstad KM, Burkett LM, Whitford WG (2009) Monitoring manual for grassland shrubland and savanna ecosystems: quick start, vol I. USDA-ARS Jornada Experimental Range, Las Cruces

Higgins SI, Bond WJ, Trollope WSW, Bondt WJ, Trollopet WSW (2000) Fire, resprouting and variability: a recipe for grass-tree coexistence in savanna. J Ecol 88:213-229

Hoffmann WA, Franco AC (2003) Comparative growth analysis of tropical forest and savanna woody plants using phylogenetically independent contrasts. J Ecol 91:475-484

Holl KD, Aide TM (2011) When and where to actively restore ecosystems? For Ecol Manag 261:1558-1563

Holl KD et al (2014) Efficacy of exotic control strategies for restoring coastal prairie grasses. Weed Sci Soc Am 7:590-598

Hulvey KB, Zavaleta ES (2012) Abundance declines of a native forb have nonlinear impacts on grassland invasion resistance. Ecology 93:378-388

INMET (2009) Normais Climatológicas Do Brasil 1961-1990. http:// www.inmet.gov.br/portal/index.php?r=clima/normaisclimatologi cas. Accessed 21 Oct 2016

Kuhlmann M (2012). Frutos E Sementes Do Cerrado Atrativos Para a Fauna: Guia de Campo. Col. Christopher W. Fagg. Rede de Sementes do Cerrado, Brasília

Kutschenko DC (2009) Fenologia E Caracterização de Frutos E Sementes de Um Cerrado Sensu Stricto, Jardim Botânico de Brasília, Distrito Federal, Brasil, Com Ênfase Nas Espécies Com Síndrome Ornitocórica. Dissertation, Universidade de Brasília, Brasília

Levine JM et al (2003) Mechanisms underlying the impacts of exotic plant invasions. Proc R Soc Lond B Biol Sci 270:775-781

Mendonça RC et al (2008) Flora Vascular Do Bioma Cerrado: Checklist Com 12.356 Espécies. In: de Almeida SP, Ribeiro JF, Sano SM (eds) Cerrado: ecologia e flora, vol 2. Embrapa Informação Tecnológica, Brasília, pp 422-442

Mori ES, Piña-Rodrigues FCM, Ivanauskas NM, Penteado de Freitas, N, Brancalion PHS, Martins RB (2012) Guia para germinação de 100 espécies nativas. In: Martins RB (org) Sementes florestais: Guia para germinação de100 espécies nativas. Instituto Refloresta, São Paulo, pp 29-141

Oliveira RS et al (2005) Deep root function in soil water dynamics in Cerrado savannas of central Brazil. Funct Ecol 19:574-581 
Overbeck GE et al (2013) Restoration ecology in Brazil-time to step out of the forest. Nat Conserv 11:92-95

Palma AC, Laurance SGW (2015) A review of the use of direct seeding and seedling plantings in restoration: what do we know and where should we go? Appl Veg Sci 18:561-568

Ratter JA, Ribeiro JF, Bridgewater S (1997) The Brazilian Cerrado vegetation and threats to its biodiversity. Ann Bot Lond 80:223-230

Rodrigues RR, Lima RAF, Gandolfi S, Nave AG (2009) On the restoration of high diversity forests: 30 years of experience in the Brazilian Atlantic forest. Biol Conserv 142:1242-1251

Ruiz-Jaen MC, Aide TM (2005) Restoration success: how is it being measured? Restor Ecol 13:569-577

Salomão AN et al (2003) Germinação de Sementes E Produção de Mudas de Plantas Do Cerrado. Rede de Sementes do Cerrado, Brasília

Sano EE, Rosa R, Brito JLS, Ferreira LG (2007) Mapeamento de cobertura cegetal do bioma Cerrado: estratégias e resultados. Embrapa Cerrados, Brasilia

Silva RPRP, Oliveira DR, da Rocha GPE, Vieira DLM (2015) Direct seeding of Brazilian savanna trees: effects of plant cover and fertilization on seedling establishment and growth. Restor Ecol 23:393-401

Soares Filho B et al (2014) Cracking Brazil's forest code. Science 344:363-364

Veldman JW et al (2015a) Toward an old-growth concept for grasslands, savannas, and woodlands. Front Ecol Environ 13:154-162

Veldman JW, Overbeck GE, Negreiros D, Mahy G, Le Stradic S, Fernandes GW, Durigan G, Buisson E, Putz F, Bond WJ (2015b) Where tree planting and forest expansion are bad for biodiversity and ecosystem services. BioSci Adv Access 20:1-8

Williams DG, Baruch Z (2000) African grass invasion in the Americas: ecosystem consequences and the role of ecophysiology. Biol Invasions 2:123-140

Young TP, Chase JM, Huddleston RT (2001) Community succession and assembly comparing, contrasting and combining paradigms in the context of ecological restoration. Ecol Restor 19:5-18

Zenni RD, Ziller SR (2011) An overview of invasive plants in Brazil. Rev Bras Bot 34:431-446 